\title{
A Novel Approach to Sliding Mode Control of Time-Delay Systems
}

\author{
Hongwei Xia, ${ }^{1} \mathrm{Li} \mathrm{Li}^{2}{ }^{2}$ Yanmin Wang, ${ }^{1}$ Aiguo $\mathrm{Wu},{ }^{3}$ and Guangcheng $\mathrm{Ma}^{1}$ \\ ${ }^{1}$ Space Control and Inertial Technology Research Center, Harbin Institute of Technology, Harbin 150001, China \\ ${ }^{2}$ School of Information Science and Electrical Engineering, Harbin Institute of Technology, Weihai 264200, China \\ ${ }^{3}$ Shenzhen Graduate School, Harbin Institute of Technology, Shenzhen 518055, China \\ Correspondence should be addressed to Hongwei Xia; xiahongwei@hit.edu.cn
}

Received 9 June 2013; Revised 29 August 2013; Accepted 29 August 2013

Academic Editor: Rongni Yang

Copyright (C) 2013 Hongwei Xia et al. This is an open access article distributed under the Creative Commons Attribution License, which permits unrestricted use, distribution, and reproduction in any medium, provided the original work is properly cited.

\begin{abstract}
This paper is concerned with the sliding mode control for a class of linear systems with time-varying delays. By utilizing a novel Lyapunov-Krasovskii functional and combining it with the delay fractioning approach as well as the free-weighting matrix technology, a sufficient condition is established such that the resulting sliding mode dynamics is asymptotically stable. Then, a sliding mode controller for reaching motion is synthesized to guarantee that the trajectories of the resulting closed-loop system can be driven onto a prescribed sliding surface and maintained there for all subsequent time. A numerical example is provided to illustrate the effectiveness of the proposed design approach.
\end{abstract}

\section{Introduction}

Since time delays often appear in various engineering, biological, and economical systems, which are frequently a source of instability or poor performance, the analysis and synthesis of time-delay systems have been one of the most active research areas in system science over the past decades; see, for example, [1-7] and the references therein. Time-delay systems are also called systems with dead-time or aftereffect, equations with deviating argument or differential-difference equations [5], belonging to the class of functional differential equations that are infinite dimensional and dependent on the past history of the dynamics [6-10]. In the literature, a great number of results have been published based on different design approaches such as characteristic equation method, Lyapunov technique, or state solution approach [8-12]. Those developed criteria can be roughly divided into two categories, namely, delay-independent stability criteria [8-10] and delaydependent stability criteria [12-18]. In general, the delaydependent stability criterion is less conservative than delayindependent one, particularly when the size of time-delay is small or time-varying [15-18].
In the process of stability analysis and synthesis, a Lyapunov function (or functional) is usually involved, and more or less tight techniques to bound some cross terms are used. Since the general form of this functional leads to a complicated system of partial differential equations [17-20], many researchers tried to construct special forms of Lyapunov functional and derived simpler sufficient conditions which are more conservative. Therefore, these choices of specific functional and overbounding techniques are the origin of conservatism $[17,18]$. In order to reduce the conservatism, some efforts were made to improve the delay-dependent conditions. For instance, the delay-partitioning idea $[18,20]$ has been proven to be an effective approach to reduce the conservatism, and many researchers have employed and further improved the idea of delay-partitioning to analyze the stability of time-delay systems. More results can be found in the literature; see, for example, [15-18] and the references therein. What is more, Wu et al. [21] and He et al. [22] have developed new methods to obtain less conservative delaydependent stability conditions. Those methods are quite different from the conventional ones of using model transformation which may introduce some superfluous dynamics 
into the original system [15-18, 21-23]. Additional studies can be found in [12-19] and references cited therein.

On the other hand, the sliding mode control (SMC) scheme is well known for its robustness against incompletely modeled dynamics, disturbances, time delays, and nonlinearities [23-25]. In the past two decades, sliding mode control has successfully been applied to a wide variety of practical engineering systems such as power systems, cantilever beam systems, spacecrafts, underwater vehicles, electrical motors, and industrial boiler systems [25-36]. Sliding mode control utilizes a discontinuous control to drive the system state trajectories to some predefined sliding surfaces on which the system has the desired properties such as stability, disturbance rejection capability, and tracking ability [23-25]. Thus, sliding mode control approach displays good performances including fast response, good transient performance, and easy realization [24]. A great volume of the literature has been published on the general topic of sliding mode control problems for various systems; see, for example, uncertain systems [30-32, 37], Markovian jump systems [24, 32], singular systems [24, 36], time-delay systems [31, 33, 35, 36], sliding mode-based observers [26, 29], stochastic systems [35], and so forth. To mention a few, Wu et al. investigated the problem of sliding mode control of Markovian jump singular timedelay systems in [24], and Khan et al. presented a dynamic integral sliding mode controller which is based on the existing dynamic sliding mode control and integral sliding mode control techniques in [37]. However, when revisiting those problems, we find that there is still much room left for improvement, which motivates the present study.

In this context, we are interested in investigating the delay-dependent sliding mode control problem of a class of time-varying delay systems. By dividing the time-delay interval into multiple segments, using the Lyapunov functional technology combined with matrix inequality technology, a new delay-dependent sufficient condition for the existence of linear sliding surfaces is proposed. To obtain a less conservative delay-dependent condition, some slack matrix variables based on the Newton-Leibniz formula are introduced. And an explicit parameterization of the desired sliding surface is also given. Then, a sliding mode controller for reaching motion is synthesized to guarantee that the trajectories of the resulting closed-loop system can be driven onto a prescribed sliding surface and maintained there for all subsequent time.

The rest of the paper is organized as follows. Section 2 gives problem formulations and some necessary lemmas. In Section 3, we present our main results of sliding mode control problem. Numerical examples are given in Section 4, followed by the conclusions, which are presented in Section 5.

Notations. The notations used throughout the paper are standard except where otherwise stated. The superscript " $T$ " stands for matrix transposition. " $I$ " and " 0 " denote the identity matrix and a zero matrix with compatible dimensions, respectively. The notation " $P>0(P \geq 0)$ " means that $P$ is real symmetric and positive definite (positive semidefinite); $\operatorname{diag}\left\{M_{1}, M_{2}, \ldots, M_{n}\right\}$ stands for a block-diagonal matrix with matrices $M_{1}, M_{2}, \ldots, M_{n}$ on the diagonal. “*” represents the elements below the main diagonal of a symmetric matrix. $\|$. || denotes the Euclidean norm of a vector or the spectral norm of a matrix. Matrices, if their dimensions are not explicitly stated, are assumed to be compatible for algebraic operations.

\section{Problem Formulations and Preliminaries}

Consider a class of linear time-delay systems given by

$$
\begin{gathered}
\dot{x}(t)=A x(t)+A_{d} x(t-d(t))+B u(t)+f(t), \quad t>0 \\
x(t)=\phi(t), \quad t \in[-h, 0],
\end{gathered}
$$

where $x(t) \in R^{n}$ is the state vector, $u(t) \in R^{m}$ is the controlled input vector, and $f(t)$ represents the neglected dynamics and external disturbance; the matrices $A, A_{d}$, and $B$ are known constant matrices of appropriate dimensions. $\phi(t)(t \in$ $[-h, 0])$ is the system's initial function which is continuously differentiable on $[-h, 0]$. Time delay $d(t)$ is a continuously differentiable function, satisfying the conditions as the following:

$$
\begin{gathered}
0 \leq d(t) \leq h \\
\dot{d}(t) \leq \mu,
\end{gathered}
$$

where $h, \mu$ are known positive real constants.

The following preliminary assumptions are made for system (1) [23].

Assumption 1. The pair $\left(A+A_{d}, B\right)$ is controllable.

Assumption 2. The perturbation term $f$ satisfies the classical matching conditions, that is,

$$
f(t)=B w(t) \quad \text { or } \quad B u(t)+f(t)=B(u(t)+w(t)),
$$

and it is bounded, as follows, by a known functional $\rho(t)$ :

$$
\|f(t)\|<\rho(t) .
$$

Assumption 3. Matrix $B$ is of full column rank; that is, rank $(B)=m$.

The aim of this paper is to design a SMC controller $u(t)$ such that the resulting closed-loop system is asymptotically stable for all exogenous disturbance. Basically, the design procedure of a sliding mode control system is a two-stage process. The first phase is to choose a set of sliding manifolds such that the original system restricted to the intersection of this sliding manifold has a desired behavior. The second phase is to design a sliding mode control law that forces the system's trajectories onto the sliding surface and maintains them on it.

We first transform the original system (1) into a special form (classically called regular form) that is appropriate for sliding mode control. A nonsingular matrix $T$ can be chosen such that

$$
T B=\left[\begin{array}{c}
0_{(n-m) \times m} \\
B_{2}
\end{array}\right],
$$


where $B_{2} \in R^{m \times m}$ is nonsingular. Thus, by a state transformation $z(t)=T x(t)$, the system (1) has the regular form

$$
\begin{gathered}
\dot{z}(t)=\bar{A} z(t)+\bar{A}_{d} z(t-d(t))+\bar{B}(u(t)+w(t)) \\
z(t)=\bar{\phi}(t), \quad t \in[-r, 0],
\end{gathered}
$$

where $\bar{A}=T A T^{-1}, \bar{A}_{d}=T A_{d} T^{-1}, \bar{B}=\left[\begin{array}{c}0_{(n-m) \times m} \\ B_{2}\end{array}\right]$, and $\bar{\phi}(t)=$ $T \phi(t)$.

Let $z(t) \triangleq\left[\begin{array}{l}z_{1}(t) \\ z_{2}(t)\end{array}\right], \bar{A} \triangleq\left[\begin{array}{ll}\bar{A}_{11} & \bar{A}_{12} \\ \bar{A}_{21} & \bar{A}_{22}\end{array}\right]$, and $\bar{A}_{d} \triangleq\left[\begin{array}{ll}\bar{A}_{d 11} & \bar{A}_{d 12} \\ \bar{A}_{d 21} & \bar{A}_{d 22}\end{array}\right]$; then system (6) can be rewritten as

$$
\begin{gathered}
\dot{z}_{1}(t)=\bar{A}_{11} z_{1}(t)+\bar{A}_{d 11} z_{1}(t-d(t)) \\
+\bar{A}_{12} z_{2}(t)+\bar{A}_{d 12} z_{2}(t-d(t)) \\
\dot{z}_{2}(t)=\bar{A}_{21} z_{1}(t)+\bar{A}_{d 21} z_{1}(t-d(t))+\bar{A}_{22} z_{2}(t) \\
+\bar{A}_{d 22} z_{2}(t-d(t))+B_{2}(u(t)+w(t)) \\
z_{1}(t)=\bar{\phi}_{1}(t) \\
z_{2}(t)=\bar{\phi}_{2}(t),
\end{gathered}
$$

where $z_{1}(t) \in R^{(n-m)}, z_{2}(t) \in R^{m}, \bar{\phi}_{1}(t) \in R^{(n-m)}$ and $\bar{\phi}_{2}(t) \epsilon$ $R^{m}$ are subblocks of $\bar{\phi}(t)$.

It is obvious that the first equation of system (7) represents the sliding motion dynamics of (6); therefore, the corresponding sliding surface can be chosen as follows:

$$
S(t)=\left[\begin{array}{ll}
C & I
\end{array}\right] z(t)=C z_{1}(t)+z_{2}(t)=0,
$$

where $C \in R^{(n-m)}$ is the parameter to be designed.

When the system trajectories reach onto the sliding surface $S(t)=\dot{S}(t)=0$, that is, $z_{2}(t)=-C z_{1}(t)$, the sliding mode dynamics is attained. By combining (7) and (8), we obtain the sliding motion

$$
\begin{gathered}
\dot{z}_{1}(t)=\left(\bar{A}_{11}-\bar{A}_{12} C\right) z_{1}(t)+\left(\bar{A}_{d 11}-\bar{A}_{d 12} C\right) z_{1}(t-d(t)), \\
z_{1}(t)=\bar{\phi}_{1}(t) .
\end{gathered}
$$

In this paper, we are interested in designing a reaching motion control law $u(t)$ such that the following requirements are satisfied.

(i) The corresponding sliding motion (9) is asymptotically stable.

(ii) The system (7) is asymptotically stable with the reaching control law $u(t)$.

To facilitate further developments, we introduce the following lemma that will be frequently used in deriving the main results.

Lemma 4 (Schur complement). For symmetrical matrix $L=$ $\left[\begin{array}{ll}L_{11} & L_{12} \\ L_{12}^{T} & L_{22}\end{array}\right]$, the following inequalities are equivalent:

(1) $L<0$,

(2) $L_{11}<0, L_{22}-L_{12}^{T} L_{11}^{-1} L_{12}<0$,

(3) $L_{22}<0, L_{11}-L_{12} L_{22}^{-1} L_{12}^{T}<0$.

\section{Main Results}

3.1. Sliding Mode Dynamics Analysis. Due to the influence of the sliding manifold on system stability and transient performance, the design and analysis of the sliding manifold has become one of the main issues in the sliding mode control [27-30], which is shown in this subsection. In the following theorem, we first present a new delay-dependent stability condition by delay partitioning approach and free-weighting matrices technique.

Theorem 5. For given positive scalars $h>0$ and $\mu>0$, the reduced order system (9) is asymptotically stable if there exist matrices $P=P^{T}>0, Q_{i}=Q_{i}^{T} \geq 0(i=1, \ldots, 4), Z=Z^{T}>0$,

$$
X=\left[\begin{array}{ccccc}
X_{11} & X_{12} & X_{13} & X_{14} & X_{15} \\
* & X_{22} & X_{23} & X_{24} & X_{25} \\
* & * & X_{33} & X_{34} & X_{35} \\
* & * & * & X_{44} & X_{45} \\
* & * & * & * & X_{55}
\end{array}\right] \geq 0
$$

and free-weighting matrices $N_{i}(i=1,2, \ldots, 5)$ with appropriate dimensions, such that the following LMIs hold:

$$
\begin{aligned}
& \Phi=\left[\begin{array}{cccccc}
\Phi_{11} & \Phi_{12} & \Phi_{13} & \Phi_{14} & \Phi_{15} & \Phi_{16} \\
* & \Phi_{22} & \Phi_{23} & \Phi_{24} & \Phi_{25} & 0 \\
* & * & \Phi_{33} & \Phi_{34} & \Phi_{35} & \Phi_{36} \\
* & * & * & \Phi_{44} & \Phi_{45} & 0 \\
* & * & * & * & \Phi_{55} & 0 \\
* & * & * & * & * & -h Z
\end{array}\right]<0, \\
& \Psi=\left[\begin{array}{cccccc}
X_{11} & X_{12} & X_{13} & X_{14} & X_{15} & N_{1} \\
* & X_{22} & X_{23} & X_{24} & X_{25} & N_{2} \\
* & * & X_{33} & X_{34} & X_{35} & N_{3} \\
* & * & * & X_{44} & X_{45} & N_{4} \\
* & * & * & * & X_{55} & N_{5} \\
* & * & * & * & * & Z
\end{array}\right] \geq 0,
\end{aligned}
$$

where

$$
\begin{aligned}
\Phi_{11}= & P\left(\bar{A}_{11}-\bar{A}_{12} C\right)+\left(\bar{A}_{11}-\bar{A}_{12} C\right)^{T} P \\
& +Q_{1}+N_{1}+N_{1}^{T}+h X_{11}, \\
\Phi_{12}= & N_{2}^{T}+h X_{12}, \\
\Phi_{13}= & P\left(\bar{A}_{d 11}-\bar{A}_{d 12} C\right)-N_{1}+N_{3}^{T}+h X_{13}, \\
\Phi_{14}= & N_{4}^{T}+h X_{14}, \quad \Phi_{15}=N_{5}^{T}+h X_{15}, \\
\Phi_{16}= & h\left(\bar{A}_{11}-\bar{A}_{12} C\right)^{T} Z, \\
\Phi_{22}= & -\left(1-\frac{\mu}{2}\right) Q_{1}+Q_{2}+h X_{22}, \\
\Phi_{23}= & -N_{2}+h X_{23}, \quad \Phi_{24}=h X_{24}, \\
\Phi_{25}= & h X_{25}, \\
\Phi_{33}= & -(1-\mu) Q_{2}+Q_{3}-N_{3}-N_{3}^{T}+h X_{33}, \\
\Phi_{34}= & -N_{4}^{T}+h X_{34}, \quad \Phi_{35}=-N_{5}^{T}+h X_{35},
\end{aligned}
$$




$$
\begin{aligned}
& \Phi_{36}=h\left(\bar{A}_{d 11}-\bar{A}_{d 12} C\right)^{T} \mathrm{Z}, \\
& \Phi_{44}=-\left(1-\frac{\mu}{2}\right) Q_{3}+Q_{4}+h X_{44}, \\
& \Phi_{45}=h X_{45}, \quad \Phi_{55}=-Q_{4}+h X_{55} .
\end{aligned}
$$

Proof. We define the following Lyapunov-Krasovskii functional

$$
V(t)=V_{1}(t)+V_{2}(t)+V_{3}(t)+V_{4}(t)+V_{5}(t)+V_{6}(t)
$$

with

$$
\begin{aligned}
& V_{1}(t)=z_{1}^{T}(t) P z_{1}(t) \\
& V_{2}(t)=\int_{t-(d(t) / 2)}^{t} z_{1}^{T}(s) Q_{1} z_{1}(s) d s \\
& V_{3}(t)=\int_{t-d(t)}^{t-(d(t) / 2)} z_{1}^{T}(s) Q_{2} z_{1}(s) d s \\
& V_{4}(t)=\int_{t-((d(t)+h) / 2)}^{t-d(t)} z_{1}^{T}(s) Q_{3} z_{1}(s) d s \\
& V_{5}(t)=\int_{t-h}^{t-((d(t)+h) / 2)} z_{1}^{T}(s) Q_{4} z_{1}(s) d s \\
& V_{6}(t)=\int_{-h}^{0} \int_{t+\theta}^{t} \dot{z}_{1}^{T}(s) Z \dot{z}_{1}(s) d s d \theta,
\end{aligned}
$$

where $P=P^{T}>0, Q_{i}=Q_{i}^{T} \geq 0(i=1, \ldots, 4)$, and $Z=Z^{T}>$ 0 are matrices to be determined. By using the Leibniz-Newton formula, one has

$$
z_{1}(t-d(t))=z_{1}(t)-\int_{t-d(t)}^{t} \dot{z}_{1}(s) d s
$$

Due to this relation, one can introduce the following zero equation:

$$
\begin{aligned}
& 2\left[z_{1}^{T}(t) N_{1}+z_{1}^{T}\left(t-\frac{d(t)}{2}\right) N_{2}+z_{1}^{T}(t-d(t)) N_{3}\right. \\
& \left.+z_{1}^{T}\left(t-\frac{d(t)+h}{2}\right) N_{4}+z_{1}^{T}(t-h) N_{5}\right] \\
& \quad \times\left[z_{1}(t)-z_{1}(t-d(t))-\int_{t-d(t)}^{t} \dot{z}_{1}(s) d s\right]=0,
\end{aligned}
$$

where $N_{i}(i=1, \ldots, 5)$ are unknown constant matrices with appropriate dimensions.

Moreover, it follows from (2) that for any appropriate dimensional matrix $X \geq 0$, (18) is always satisfied as follows:

$$
h \eta^{T}(t) X \eta(t)-\int_{t-d(t)}^{t} \eta^{T}(t) X \eta(t) d s \geq 0,
$$

where

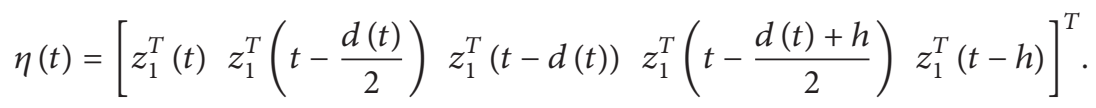

According to (16)-(19), and by taking the time derivative of the Lyapunov-Krasovskii functional in (14), we have

$\dot{V}(t)$

$$
\begin{aligned}
= & 2 z_{1}^{T}(t) P \dot{z}_{1}(t)+z_{1}^{T}(t) Q_{1} z_{1}(t) \\
& -\left(1-\frac{\dot{d}(t)}{2}\right) z_{1}^{T}\left(t-\frac{d(t)}{2}\right) Q_{1} z_{1}\left(t-\frac{d(t)}{2}\right) \\
& +\left(1-\frac{\dot{d}(t)}{2}\right) z_{1}^{T}\left(t-\frac{d(t)}{2}\right) Q_{2} z_{1}\left(t-\frac{d(t)}{2}\right) \\
& -(1-\dot{d}(t)) z_{1}^{T}(t-d(t)) Q_{2} z_{1}(t-d(t)) \\
& +(1-\dot{d}(t)) z_{1}^{T}(t-d(t)) Q_{3} z_{1}(t-d(t)) \\
& -\left(1-\frac{\dot{d}(t)}{2}\right) z_{1}^{T}\left(t-\frac{d(t)+h}{2}\right) Q_{3} z_{1}\left(t-\frac{d(t)+h}{2}\right)
\end{aligned}
$$

$$
\begin{aligned}
& +\left(1-\frac{\dot{d}(t)}{2}\right) z_{1}^{T}\left(t-\frac{d(t)+h}{2}\right) Q_{4} z_{1}\left(t-\frac{d(t)+h}{2}\right) \\
& -z_{1}^{T}(t-h) Q_{4} z_{1}(t-h)+h \dot{z}_{1}^{T}(t) Z_{z_{1}}(t) \\
& -\int_{t-h}^{t} \dot{z}_{1}^{T}(s) Z \dot{z}_{1}(s) d s \\
& \leq 2 z_{1}^{T}(t) P\left(\bar{A}_{11}-\bar{A}_{12} C\right) z_{1}(t) \\
& +\left(\bar{A}_{d 11}-\bar{A}_{d 12} C\right) z_{1}(t-d(t))+z_{1}^{T}(t) Q_{1} z_{1}(t) \\
& -\left(1-\frac{\mu}{2}\right) z_{1}^{T}\left(t-\frac{d(t)}{2}\right) Q_{1} z_{1}\left(t-\frac{d(t)}{2}\right) \\
& +\left(1-\frac{\dot{d}(t)}{2}\right) z_{1}^{T}\left(t-\frac{d(t)}{2}\right) Q_{2} z_{1}\left(t-\frac{d(t)}{2}\right) \\
& -(1-\mu) z_{1}^{T}(t-d(t)) Q_{2} z_{1}(t-d(t))
\end{aligned}
$$




$$
\begin{aligned}
& +(1-\dot{d}(t)) z_{1}^{T}(t-d(t)) Q_{3} z_{1}(t-d(t)) \\
& -\left(1-\frac{\mu}{2}\right) z_{1}^{T}\left(t-\frac{d(t)+h}{2}\right) Q_{3} z_{1}\left(t-\frac{d(t)+h}{2}\right) \\
& +\left(1-\frac{\dot{d}(t)}{2}\right) z_{1}^{T}\left(t-\frac{d(t)+h}{2}\right) Q_{4} z_{1}\left(t-\frac{d(t)+h}{2}\right) \\
& -z_{1}^{T}(t-h) Q_{4} z_{1}(t-h)+h \dot{z}_{1}^{T}(t) Z_{1}(t) \\
& -\int_{t-d(t)}^{t} \dot{z}_{1}^{T}(s) Z \dot{z}_{1}(s) d s \\
& +2\left[z_{1}^{T}(t) N_{1}+z_{1}^{T}\left(t-\frac{d(t)}{2}\right) N_{2}+z_{1}^{T}(t-d(t)) N_{3}\right. \\
& \left.+z_{1}^{T}\left(t-\frac{d(t)+h}{2}\right) N_{4}+z_{1}^{T}(t-h) N_{5}\right] \\
& +\left[z_{1}(t)-z_{1}(t-d(t))-\int_{t-d(t)}^{t} \dot{z}_{1}(s) d s\right] \\
& =\eta^{T}(t) \Xi \eta(t)-\int_{t-d(t)}^{t} \xi^{T}(t, s) \Psi \xi(t, s) d s-\Upsilon \\
& \eta^{T}(t) X \eta(t) d s \\
& +\int_{t}^{t}(t)
\end{aligned}
$$

where

$$
\begin{aligned}
& \xi(t)=\left[\begin{array}{ll}
\eta^{T}(t) & \dot{z}_{1}^{T}(s)
\end{array}\right]^{T}, \\
& \Upsilon=\frac{\dot{d}(t)}{2} z_{1}^{T}\left(t-\frac{d(t)}{2}\right) Q_{2} z_{1}\left(t-\frac{d(t)}{2}\right) \\
& +\dot{d}(t) z_{1}^{T}(t-d(t)) Q_{3} z_{1}(t-d(t)) \\
& +\frac{\dot{d}(t)}{2} z_{1}^{T}\left(t-\frac{d(t)+h}{2}\right) Q_{4} z_{1}\left(t-\frac{d(t)+h}{2}\right), \\
& \Xi=\left[\begin{array}{ccccc}
\Phi_{11} & \Phi_{12} & \bar{\Phi}_{13} & \Phi_{14} & \Phi_{15} \\
* & \Phi_{22} & \Phi_{23} & \Phi_{24} & \Phi_{25} \\
* & * & \Phi_{33} & \Phi_{34} & \Phi_{35} \\
* & * & * & \Phi_{44} & \Phi_{45} \\
* & * & * & * & \Phi_{55}
\end{array}\right], \\
& \bar{\Phi}_{11}=\Phi_{11}+\left(\bar{A}_{11}-\bar{A}_{12} C\right)^{T} H\left(\bar{A}_{11}-\bar{A}_{12} C\right), \\
& \bar{\Phi}_{13}=\Phi_{13}+\left(\bar{A}_{11}-\bar{A}_{12} C\right)^{T} H\left(\bar{A}_{d 11}-\bar{A}_{d 12} C\right), \\
& \bar{\Phi}_{33}=\Phi_{33}+\left(\bar{A}_{d 11}-\bar{A}_{d 12} C\right)^{T} H\left(\bar{A}_{d 11}-\bar{A}_{d 12} C\right)
\end{aligned}
$$

and $\Psi$ is denoted in (12).
By Schur complement (Lemma 4) and some matrices primary manipulations, it is easy to conclude that the abovementioned matrix $\Xi<0$ in (21) is equivalent to (11). Since $Q_{2} \geq 0, Q_{3} \geq 0$, and $Q_{4} \geq 0$, we have $\Upsilon \geq 0$. Therefore, if $\Xi<0$ and $\Psi \geq 0$, there exists a positive scalar $\varepsilon$ such that $\dot{V}(t) \leq-\varepsilon\left\|z_{1}(t)\right\|^{2}$, which guarantees that system (7) is asymptotically stable.

Thus, the proof is completed.

Remark 6. It should be pointed out that Theorem 5 presents a delay-dependent admissible criterion for sliding mode dynamics by utilizing a novel Lyapunov-Krasovskii functional (LKF), which is much less conservative than mos results in the literature. The reduced conservatism of Theorem 5 benefits from the construction of the new LKF in (14) based on the idea of delay partitioning. In the general delay-partitioning method, the time delay should be modeled and represented as a constant part and a time-varying part, and then, the idea of delay partitioning is applied to the constant part [16-19]. However, it is not suitable to the case when the time delay cannot be modeled as those two parts. According to this, in this brief, we divided the whole time varying delay interval $[0, h]$ into multiparts

$$
\begin{array}{cc}
{\left[0, \frac{d(t)}{2}\right],} & {\left[\frac{d(t)}{2}, d(t)\right],} \\
{\left[d(t), \frac{d(t)+h}{2}\right],} & {\left[\frac{d(t)+h}{2}, h\right],}
\end{array}
$$

and a novel LKF is constructed with every delay component. Thus, we obtain a time varying LKF, since it is dependent on the time varying delay, which constitutes the major difference from most existing results in the literature.

Remark 7. Note that the criteria for sliding mode dynamics analysis problem in Theorem 5 are not LMI conditions due to the term $C^{T} \bar{A}_{12}^{T} \mathrm{Z}$ in (11). Then, based on Theorem 5, by carrying out some algebraic manipulations, we can obtain the following condition which is characterized in terms of a set of linear matrix inequalities and can be easily solved by using the existing Matlab LMI toolbox.

Theorem 8. For given positive scalars $h>0$ and $\mu>0$, the reduced order system (9) is asymptotically stable if there exist matrices $\widetilde{P}=\widetilde{P}^{T}>0, \widetilde{Q}_{i}=\widetilde{Q}_{i}^{T} \geq 0(i=1, \ldots, 4), \widetilde{Z}=\widetilde{Z}^{T}>0$,

$$
\widetilde{X}=\left[\begin{array}{ccccc}
\widetilde{X}_{11} & \widetilde{X}_{12} & \widetilde{X}_{13} & \widetilde{X}_{14} & \widetilde{X}_{15} \\
* & \widetilde{X}_{22} & \widetilde{X}_{23} & \widetilde{X}_{24} & \widetilde{X}_{25} \\
* & * & \widetilde{X}_{33} & \widetilde{X}_{34} & \widetilde{X}_{35} \\
* & * & * & \widetilde{X}_{44} & \widetilde{X}_{45} \\
* & * & * & * & \widetilde{X}_{55}
\end{array}\right] \geq 0
$$


and free-weighting matrices $\widetilde{N}_{i}(i=1, \ldots, 5)$ with appropriate dimensions, such that the following LMIs hold:

$$
\begin{aligned}
& \widetilde{\Phi}=\left[\begin{array}{cccccc}
\widetilde{\Phi}_{11} & \widetilde{\Phi}_{12} & \widetilde{\Phi}_{13} & \widetilde{\Phi}_{14} & \widetilde{\Phi}_{15} & \widetilde{\Phi}_{16} \\
* & \widetilde{\Phi}_{22} & \widetilde{\Phi}_{23} & \widetilde{\Phi}_{24} & \widetilde{\Phi}_{25} & 0 \\
* & * & \widetilde{\Phi}_{33} & \widetilde{\Phi}_{34} & \widetilde{\Phi}_{35} & \widetilde{\Phi}_{36} \\
* & * & * & \widetilde{\Phi}_{44} & \widetilde{\Phi}_{45} & 0 \\
* & * & * & * & \Phi_{55} & 0 \\
* & * & * & * & * & \widetilde{\Phi}_{66}
\end{array}\right]<0, \\
& \widetilde{\Psi}=\left[\begin{array}{cccccc}
\widetilde{X}_{11} & \widetilde{X}_{12} & \widetilde{X}_{13} & \widetilde{X}_{14} & \widetilde{X}_{15} & \widetilde{N}_{1} \\
* & \widetilde{X}_{22} & \widetilde{X}_{23} & \widetilde{X}_{24} & \widetilde{X}_{25} & \widetilde{N}_{2} \\
* & * & \widetilde{X}_{33} & \widetilde{X}_{34} & \widetilde{X}_{35} & \widetilde{N}_{3} \\
* & * & * & \widetilde{X}_{44} & \widetilde{X}_{45} & \widetilde{N}_{4} \\
* & * & * & * & \widetilde{X}_{55} & \widetilde{N}_{5} \\
* & * & * & * & * & \widetilde{Z}
\end{array}\right] \geq 0,
\end{aligned}
$$

where

$$
\begin{aligned}
\widetilde{\Phi}_{11}= & \left(\bar{A}_{11} \widetilde{P}-\bar{A}_{12} \widetilde{C}\right)+\left(\bar{A}_{11} \widetilde{P}-\bar{A}_{12} \widetilde{C}\right)^{T} \\
& +\widetilde{Q}_{1}+\widetilde{N}_{1}+\widetilde{N}_{1}^{T}+h \widetilde{X}_{11}, \\
\widetilde{\Phi}_{12}= & \widetilde{N}_{2}^{T}+h \widetilde{X}_{12}, \\
\widetilde{\Phi}_{13}= & \left(\bar{A}_{d 11} \widetilde{P}-\bar{A}_{d 12} \widetilde{C}\right)-\widetilde{N}_{1}+\widetilde{N}_{3}^{T}+h \widetilde{X}_{13}, \\
\widetilde{\Phi}_{14}= & \widetilde{N}_{4}^{T}+h \widetilde{X}_{14}, \quad \widetilde{\Phi}_{15}=\widetilde{N}_{5}^{T}+h \widetilde{X}_{15}, \\
\widetilde{\Phi}_{16}= & \left(\bar{A}_{11} \widetilde{P}-\bar{A}_{12} \widetilde{C}\right)^{T}, \\
\widetilde{\Phi}_{22}= & -\left(1-\frac{\mu}{2}\right) \widetilde{Q}_{1}+\widetilde{Q}_{2}+h \widetilde{X}_{22}, \\
\widetilde{\Phi}_{23}= & -\widetilde{N}_{2}+h \widetilde{X}_{23}, \quad \widetilde{\Phi}_{24}=h \widetilde{X}_{24}, \\
\widetilde{\Phi}_{25}= & h \widetilde{X}_{25}, \\
\widetilde{\Phi}_{33}= & -(1-\mu) \widetilde{Q}_{2}+\widetilde{Q}_{3}-\widetilde{N}_{3}-\widetilde{N}_{3}^{T}+h \widetilde{X}_{33}, \\
\widetilde{\Phi}_{34}= & -\widetilde{N}_{4}^{T}+h \widetilde{X}_{34}, \quad \\
\widetilde{\Phi}_{35}= & -\widetilde{N}_{5}^{T}+h \widetilde{X}_{35}, \quad \widetilde{\Phi}_{36}=\left(\bar{A}_{d 11} \widetilde{P}-\bar{A}_{d 12} \widetilde{C}\right)^{T}, \\
\widetilde{\Phi}_{44}= & -\left(1-\frac{\mu}{2}\right) \widetilde{Q}_{3}+\widetilde{Q}_{4}+h \widetilde{X}_{44}, \\
\widetilde{\Phi}_{45}= & h \widetilde{X}_{45}, \quad \widetilde{\Phi}_{55}=-\widetilde{Q}_{4}+h \widetilde{X}_{55}, \\
\widetilde{\Phi}_{66}= & h{ }^{-1}(\widetilde{Z}-2 \widetilde{P}) .
\end{aligned}
$$

Moreover, if the conditions mentioned above are feasible, the matrix $C$ in (8) can be given by $C=\widetilde{C} \widetilde{P}^{-1}$; that is, the sliding surface of (7) can be designed as

$$
S(t)=\left(\widetilde{C} \widetilde{P}^{-1}\right) z_{1}(t)+z_{2}(t)=0 .
$$

Proof. By performing a congruence transformation diag $\left\{P^{-1}, P^{-1}, P^{-1}, P^{-1}, P^{-1}\right\}$ to both sides of (10), and defining matrices $\widetilde{X}_{m n} \triangleq P^{-1} X_{m n} P^{-1}(m, n=1,2,3,4,5)$, thus, (23) is obtained.

Similarly, performing a congruence transformation to (12) by $\operatorname{diag}\left\{P^{-1}, P^{-1}, P^{-1}, P^{-1}, P^{-1}, P^{-1}\right\}$ and defining matrices $\widetilde{N}_{k} \triangleq P^{-1} N_{k} P^{-1},(k=1,2,3,4,5), \widetilde{Z} \triangleq P^{-1} Z P^{-1}$, we have (25).

Performing a congruence transformation to (11) with $\operatorname{diag}\left\{P^{-1}, P^{-1}, P^{-1}, P^{-1}, P^{-1}, Z^{-1}\right\}$ and defining matrices $\widetilde{P} \triangleq$ $P^{-1}, \widetilde{Q}_{i} \triangleq P^{-1} Q_{i} P^{-1},(i=1,2,3,4), \widetilde{C}=C P^{-1}$, we have

$\widetilde{\Phi}$

$$
=\left[\begin{array}{cccccc}
\widetilde{\Phi}_{11} & \widetilde{\Phi}_{12} & \widetilde{\Phi}_{13} & \widetilde{\Phi}_{14} & \widetilde{\Phi}_{15} & h\left(\bar{A}_{11} \widetilde{P}-\bar{A}_{12} \widetilde{C}\right)^{T} \\
* & \widetilde{\Phi}_{22} & \widetilde{\Phi}_{23} & \widetilde{\Phi}_{24} & \widetilde{\Phi}_{25} & 0 \\
* & * & \widetilde{\Phi}_{33} & \widetilde{\Phi}_{34} & \widetilde{\Phi}_{35} & h\left(\bar{A}_{d 11} \widetilde{P}-\bar{A}_{d 12} \widetilde{C}\right)^{T} \\
* & * & * & \widetilde{\Phi}_{44} & \widetilde{\Phi}_{45} & 0 \\
* & * & * & * & \widetilde{\Phi}_{55} & 0 \\
* & * & * & * & * & -h \widetilde{P} \widetilde{Z}^{-1} \widetilde{P}
\end{array}\right]<0 .
$$

According to the definition of $\widetilde{P}$ and $\widetilde{Z}$, the following equation always holds:

$$
(\widetilde{P}-\widetilde{Z})^{T} \widetilde{Z}^{-1}(\widetilde{P}-\widetilde{Z}) \geq 0
$$

which implies that

$$
-\widetilde{P} \widetilde{Z}^{-1} \widetilde{P} \leq \widetilde{Z}-2 \widetilde{P} .
$$

Thus, we can obtain (24).

Hence, the proof is completed.

After switching surface design, the next important aspect of sliding mode control is to guarantee the existence of a sliding mode.

3.2. Sliding Mode Controller Design. Now, we are in the position to design a SMC law, by which the trajectories of the time-delay system (7) can be driven onto the predesigned sliding surface $S(t)=0$ in a finite time.

Theorem 9. Suppose that the conditions of (23)-(25) in Theorem 8 are feasible and the linear sliding surface is given by (8). Then, the trajectory of the closed-loop system (7) can be driven onto the sliding surface in limited time with the control:

$$
\begin{gathered}
u(t)=u_{e q}(t)+u_{N}(t) \\
u_{e q}(t)=-B_{2}^{-1}\left[\overline{C A} z(t)+\overline{C A}_{d} z(t-d(t))\right] \\
u_{N}(t)=-[\rho(t)+\varepsilon] \operatorname{sgn}\left(B_{2}^{T} s(t)\right),
\end{gathered}
$$

where $\varepsilon>0$ and $\rho(t)$ is the upper bound of $w(t)$.

Proof. We will complete the proof by showing that the control law (31) can not only drive the system trajectories onto the liner sliding surface but also keep it there for all subsequent time. 
According to SMC theory, when the system trajectories reach onto the sliding surface, it follows that $S(t)=0$ and $\dot{S}(t)=0$.

From the sliding surface $S=\left[\begin{array}{ll}C & I\end{array}\right] z=\bar{C} z$, we have

$$
\begin{aligned}
\dot{S} & =\left[\begin{array}{ll}
C & I
\end{array}\right] \dot{z}=\bar{C}\left(\dot{z}_{1}+\dot{z}_{2}\right) \\
& =\overline{C A} z(t)+\overline{C A}_{d} z(t-d(t))+B_{2}(u(t)+w(t)) .
\end{aligned}
$$

Therefore, by $\dot{S}(t)=0$, we get the equivalent control as follows:

$$
u_{\mathrm{eq}}(t)=-B_{2}^{-1}\left[\overline{C A} z(t)+\overline{C A}_{d} z(t-d(t))\right] .
$$

Choose the following Lyapunov functional

$$
V(t)=\frac{1}{2} S^{T}(t) S(t) .
$$

By taking the derivative of $V$, it gives

$\dot{V}(t)$

$$
\begin{aligned}
& =S^{T}(t) \dot{S}(t) \\
& =S^{T}(t)\left[\overline{C A} z(t)+\overline{C A}_{d} z(t-d(t))+B_{2}(u(t)+w(t))\right] .
\end{aligned}
$$

Combining (31) and (35), we have

$$
\begin{aligned}
\dot{V}(t) & =S^{T}(t)\left[B_{2}\left(u_{N}(t)+w(t)\right)\right] \\
& =S^{T}(t) B_{2}\left[-(\rho(t)+\varepsilon) \operatorname{sgn}\left(B_{2}^{T} S(t)\right)+w(t)\right] \\
& =-\varepsilon\left\|B_{2}^{T} S(t)\right\|-S^{T}(t) B_{2}\left(\rho(t) \operatorname{sgn}\left(B_{2}^{T} S(t)\right)-w(t)\right) \\
& \leq-\varepsilon\left\|B_{2}^{T} S(t)\right\|<0
\end{aligned}
$$

which implies that the trajectories of (7) can be driven onto the predefined sliding surface in a finite time by the control law (31) and be maintained there for all subsequent time. Thus, the proof is completed.

\section{Numerical Example}

In this section, a simulation example is used to demonstrate the effectiveness of our proposed theoretical results. Consider the following time-delay system (1) with

$$
A=\left[\begin{array}{cc}
-4 & 1.5 \\
4 & -2
\end{array}\right], \quad A_{d}=\left[\begin{array}{ll}
0.7 & 0.8 \\
0.6 & 0.9
\end{array}\right], \quad B=\left[\begin{array}{l}
1 \\
0
\end{array}\right]
$$

and $w(t)=\sin (t)$, the time delay in system (1) is selected as $h=0.8, \mu=0.2$. It is easy to check that the above system with $u(t)=0$ is unstable, and the states of the open-loop system are shown in Figure 1 with the initial condition given by $\phi(t)=\left[\begin{array}{ll}-3 & 2\end{array}\right]^{T}\left(t \in\left[\begin{array}{ll}-h & 0\end{array}\right]\right)$. Therefore, we aim to design a sliding mode controller $u(t)$ such that the closedloop system is asymptotically stable. To check the stability of

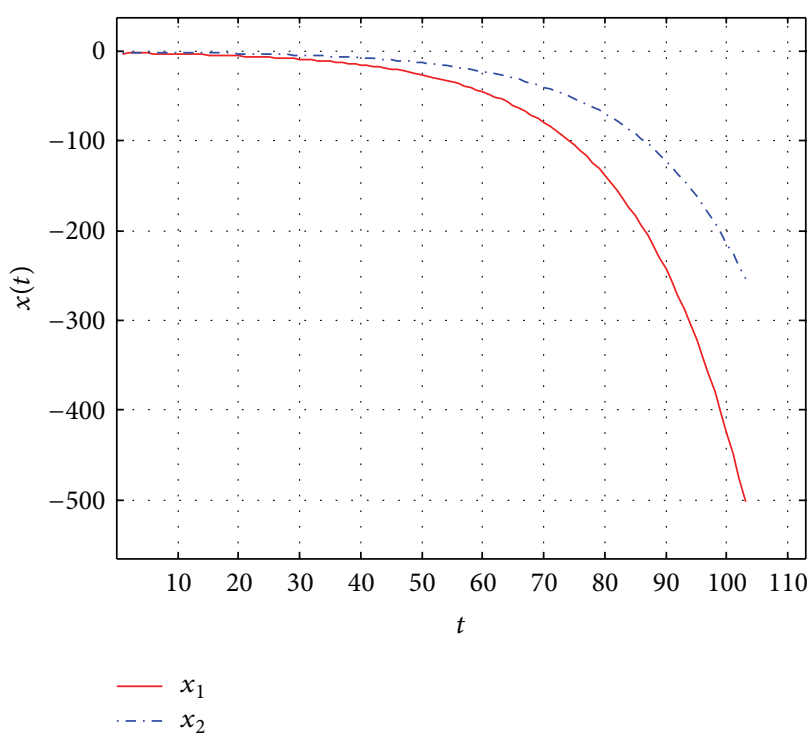

FIGURE 1: States of the open-loop system.

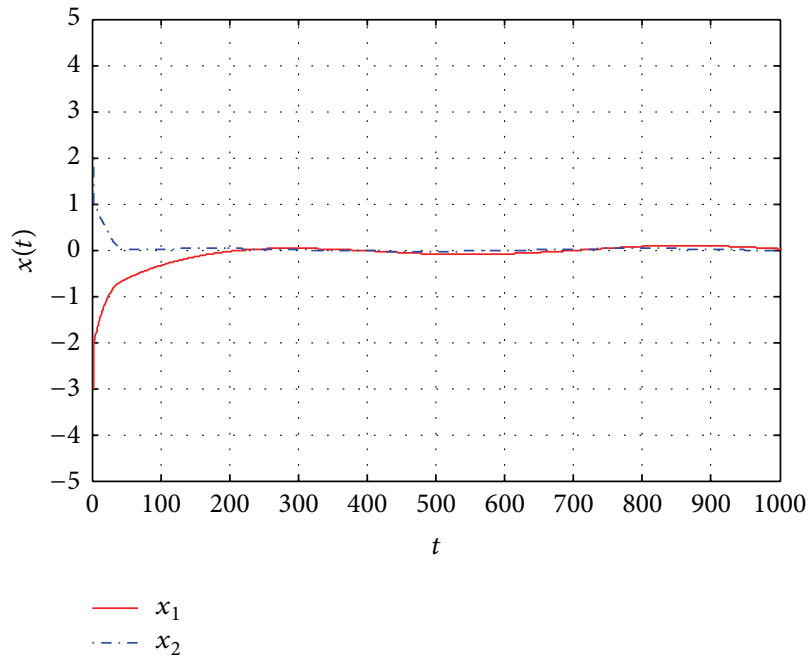

Figure 2: States of the closed-loop system.

(9), we solve conditions (23)-(25) in Theorem 8 , which gives that

$$
C=0.0384 \text {. }
$$

According to (27), we have

$$
S(t)=\left[\begin{array}{ll}
0.0384 & 1
\end{array}\right] z(t) .
$$

The existence of a feasible solution shows that there exists a delay-dependent LPK functional for checking the stability of the sliding mode dynamics in (9); that is, we can find a desired sliding surface in (39) such that the corresponding sliding mode dynamics in (9) is asymptotically stable. The remaining task is to design a sliding mode controller such that the system trajectories can be driven onto the predefined sliding surface in (39) and maintained there for all subsequent time. 


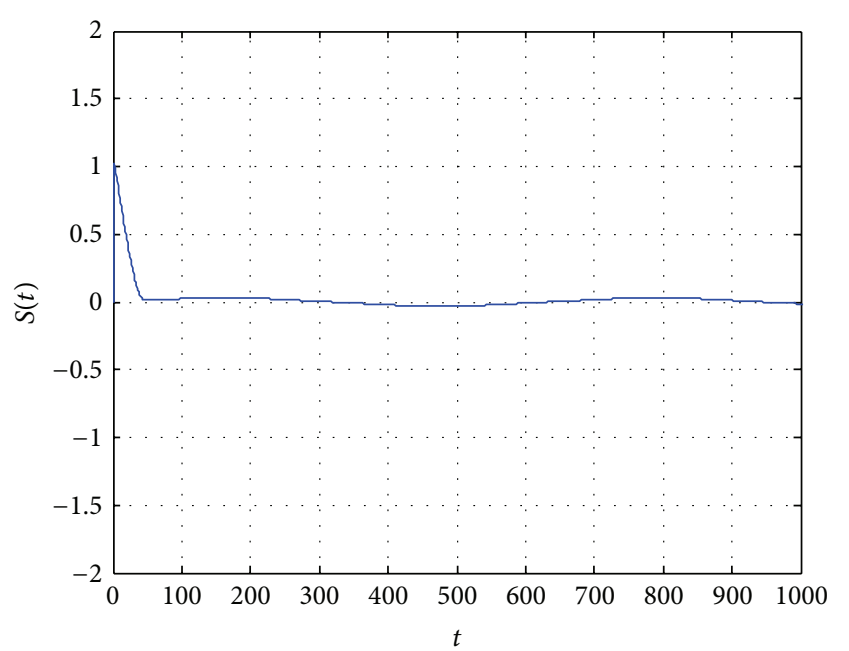

$-S$

Figure 3: Sliding surface function.

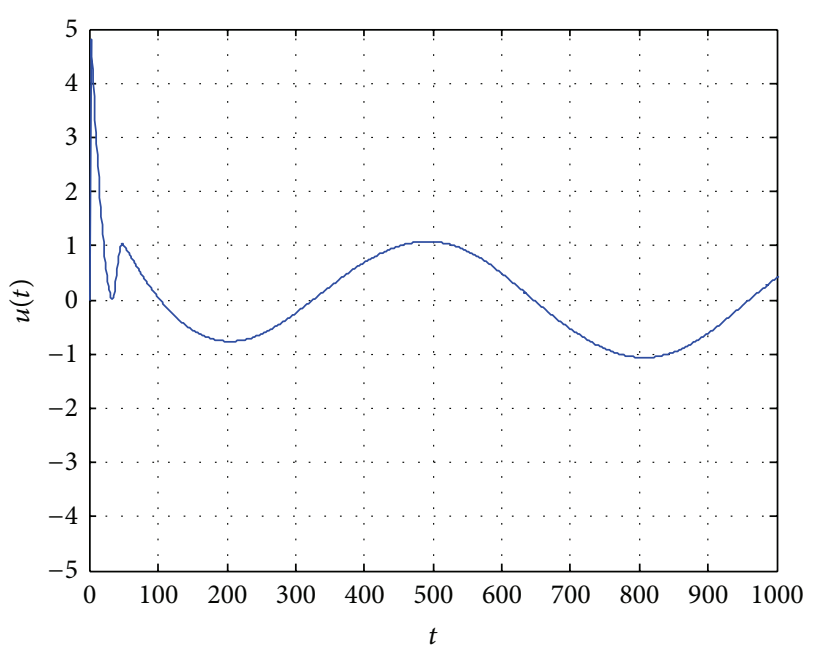

Figure 4: Control input.

According to Theorem 9, the sliding mode controller in (31) can be designed as

$$
\begin{aligned}
u(t)= & -\left[\left[\begin{array}{c}
1.4232 \\
-3.8464
\end{array}\right] z(t)+\left[\begin{array}{l}
0.8346 \\
0.7230
\end{array}\right] z(t-d(t))\right] \\
& -(\rho(t)+\varepsilon) \times \operatorname{sgn}(s(t)) .
\end{aligned}
$$

Set $\varepsilon=2, \rho(t)=1$. To prevent the control signals from chattering, we replace $\operatorname{sgn}\left(B_{2}^{T} S(t)\right)$ with $B_{2}^{T} S(t) /\left(\left\|B_{2}^{T} S(t)\right\|+\right.$ 0.01 ).

The simulation results of the closed-loop system with (40) are illustrated in Figures 2-4 with the initial condition $\phi(t)=\left[\begin{array}{ll}-3 & 2\end{array}\right]^{T}\left(t \in\left[\begin{array}{ll}-h & 0\end{array}\right]\right)$. Among them, Figure 2 shows the states responses of system (1) under the presented SMC law. The sliding surface $S(t)$ and control input signal $u(t)$ are given in Figures 3 and 4, respectively. From the simulation, it is shown that the designed SMC scheme in this paper is feasible and effective since the resulting closed-loop system is stable.

\section{Conclusion}

The problem of sliding mode control for time-delay systems has been studied in this paper. The main contribution of this paper lies in that a novel Lyapunov-Krasovskii functional that reflects the delay-fractioning nature is constructed in order to reduce the possible conservatism introduced by the time delays. It should be pointed out that the delay partitioning technique of this paper is different from others in the literature, and one of the advantages of the method is that it is not necessary to represent the time delay model with two parts, the constant part and the time varying part. Firstly, by constructing a novel Lyapunov-Krasovskii functional based on the delay partitioning approach, a sufficient condition is given to guarantee the asymptotic stability of the sliding mode dynamics. Furthermore, a sliding mode control law is proposed to ensure the reachability of the system's trajectories to the predefined sliding surface. Finally, a numerical example has been provided to demonstrate the effectiveness of the proposed methods.

\section{Acknowledgments}

The work is supported by National Natural Science Foundation of China (NSFC 61304108, NSFC 51307035, NSFC 61273094) and the Project for Distinguished Young Scholars of the Basic Research Plan in Shenzhen City under Contract no. JCJ201110001. The authors are very thankful to the reviewers for their valuable suggestions and comments.

\section{References}

[1] J. J. Batzel and F. Kappel, "Time delay in physiological systems: analyzing and modeling its impact," Mathematical Biosciences, vol. 234, no. 2, pp. 61-74, 2011.

[2] L. Wu and Z. Wang, "Guaranteed cost control of switched systems with neutral delay via dynamic output feedback," International Journal of Systems Science, vol. 40, no. 7, pp. 717728, 2009.

[3] P. Pepe and E. I. Verriest, "Lyapunov criteria for stability in $L_{p}$ norm of special neutral systems," Automatica, vol. 48, no. 1, pp. 132-138, 2012.

[4] R. Yang, P. Shi, G.-P. Liu, and H. Gao, "Network-based feedback control for systems with mixed delays based on quantization and dropout compensation," Automatica, vol. 47, no. 12, pp. 2805-2809, 2011.

[5] A. Rodkina and M. Basin, "On delay-dependent stability for a class of nonlinear stochastic delay-differential equations," Mathematics of Control, Signals, and Systems, vol. 18, no. 2, pp. 187-197, 2006.

[6] L. Wu and W. X. Zheng, "Weighted $H_{\infty}$ model reduction for linear switched systems with time-varying delay," Automatica, vol. 45, no. 1, pp. 186-193, 2009.

[7] M. T. Alrifai, M. Zribi, M. Rayan, and M. S. Mahmoud, "On the control of time delay power systems," International Journal of 
Innovative Computing, Information and Control, vol. 9, no. 2, pp. 769-792, 2013.

[8] F. O. Souza, M. C. de Oliveira, and R. M. Palhares, "Stability independent of delay using rational functions," Automatica, vol. 45, no. 9, pp. 2128-2133, 2009.

[9] S. Hirche, T. Matiakis, and M. Buss, "A distributed controller approach for delay-independent stability of networked control systems," Automatica, vol. 45, no. 8, pp. 1828-1836, 2009.

[10] P.-A. Bliman, "LMI characterization of the strong delayindependent stability of linear delay systems via quadratic Lyapunov-Krasovskii functionals," Systems \& Control Letters, vol. 43 , no. 4 , pp. 263-274, 2001.

[11] A. Seuret and F. Gouaisbaut, "Wirtinger-based integral inequality: application to time-delay systems," Automatica, vol. 49, no. 9, pp. 2860-2866, 2013.

[12] S. Xu, J. Lam, and Y. Zou, "New results on delay-dependent robust $H_{\infty}$ control for systems with time-varying delays," Automatica, vol. 42, no. 2, pp. 343-348, 2006.

[13] R. Yang, Z. Zhang, and P. Shi, "Exponential stability on stochastic neural networks with discrete interval and distributed delays," IEEE Transactions on Neural Networks, vol. 21, no. 1, pp. 169-175, 2010.

[14] N. Bekiaris-Liberis and M. Krstic, "Compensation of statedependent input delay for nonlinear systems," IEEE Transactions on Automatic Control, vol. 58, no. 2, pp. 275-289, 2013.

[15] L. Wu, X. Su, P. Shi, and J. Qiu, "A new approach to stability analysis and stabilization of discrete-time T-S fuzzy timevarying delay systems," IEEE Transactions on Systems, Man, and Cybernetics B, vol. 41, no. 1, pp. 273-286, 2011.

[16] D. Han and L. Shi, "Guaranteed cost control of affine nonlinear systems via partition of unity method," Automatica, vol. 49, no. 2, pp. 660-666, 2013.

[17] L. Wu, X. Su, P. Shi, and J. Qiu, "Model approximation for discrete-time state-delay systems in the T-S fuzzy framework," IEEE Transactions on Fuzzy Systems, vol. 19, no. 2, pp. 366-378, 2011.

[18] O. M. Kwon, M. J. Park, J. H. Park, S. M. Lee, and E. J. Cha, "New delay-partitioning approaches to stability criteria for uncertain neutral systems with time-varying delays," Journal of the Franklin Institute, vol. 349, no. 9, pp. 2799-2823, 2012.

[19] F. Gouaisbaut, M. Dambrine, and J. P. Richard, "Robust control of delay systems: a sliding mode control design via LMI," Systems \& Control Letters, vol. 46, no. 4, pp. 219-230, 2002.

[20] X. Yu, C. Wu, F. Liu, and L. Wu, "Sliding mode control of discrete-time switched systems with time-delay," Journal of the Franklin Institute, vol. 350, no. 1, pp. 19-33, 2013.

[21] M. Wu, Y. He, and J.-H. She, "New delay-dependent stability criteria and stabilizing method for neutral systems," IEEE Transactions on Automatic Control, vol. 49, no. 12, pp. 22662271, 2004.

[22] Y. He, Q.-G. Wang, L. Xie, and C. Lin, "Further improvement of free-weighting matrices technique for systems with timevarying delay," IEEE Transactions on Automatic Control, vol. 52, no. 2, pp. 293-299, 2007.

[23] J. Zhang, P. Shi, and Y. Xia, "Robust adaptive sliding-mode control for fuzzy systems with mismatched uncertainties," IEEE Transactions on Fuzzy Systems, vol. 18, no. 4, pp. 700-711, 2010.

[24] L. Wu, X. Su, and P. Shi, "Sliding mode control with bounded $L_{2}$ gain performance of Markovian jump singular time-delay systems," Automatica, vol. 48, no. 8, pp. 1929-1933, 2012.
[25] T.-C. Lin, S.-W. Chang, and C.-H. Hsu, "Robust adaptive fuzzy sliding mode control for a class of uncertain discrete-time nonlinear systems," International Journal of Innovative Computing, Information and Control, vol. 8, no. 1, pp. 347-359, 2012.

[26] A. Cid-Pastor, L. Martinez-Salamero, A. El Aroudi, R. Giral, J. Calvente, and R. Leyva, "Synthesis of loss-free resistors based on sliding-mode control and its applications in power processing," Control Engineering Practice, vol. 21, no. 5, pp. 689-699, 2013.

[27] H. Moradi, F. Bakhtiari-Nejad, M. Saffar-Avval, and A. Alasty, "Using sliding mode control to adjust drum level of a boiler unit with time varying parameters," in Proceedings of the ASME10th Biennial Conference on Engineering Systems Design and Analysis (ESDA '10), pp. 29-33, Istanbul, Turkey, July 2010.

[28] M. Vagia, "A frequency independent approximation and a sliding mode control scheme for a system of a micro-cantilever beam," ISA Transactions, vol. 51, no. 2, pp. 325-332, 2012.

[29] H. Amimeur, D. Aouzellag, R. Abdessemed, and K. Ghedamsi, "Sliding mode control of a dual-stator induction generator for wind energy conversion systems," International Journal of Electrical Power and Energy Systems, vol. 42, no. 1, pp. 60-70, 2012.

[30] S. Dadras and H. R. Momeni, "Fractional terminal sliding mode control design for a class of dynamical systems with uncertainty," Communications in Nonlinear Science and Numerical Simulation, vol. 17, no. 1, pp. 367-377, 2012.

[31] Y. Xia and Y. Jia, "Robust sliding-mode control for uncertain time-delay systems: an LMI approach," IEEE Transactions on Automatic Control, vol. 48, no. 6, pp. 1086-1092, 2003.

[32] J. Hu, Z. Wang, Y. Niu, and H. Gao, "Sliding mode control for uncertain discrete-time systems with Markovian jumping parameters and mixed delays," Journal of the Franklin Institute, 2012.

[33] A. Khandekar, G. Malwatkar, and B. Patre, "Discrete sliding mode control for robust tracking of higher order delay time systems with experimental application," ISA Transactions, vol. 52, no. 1, pp. 36-44, 2013.

[34] J. Wang and Z. Sun, "6-DOF robust adaptive terminal sliding mode control for spacecraft formation flying," Acta Astronautica, vol. 73, pp. 76-87, 2012.

[35] M. Liu and G. Sun, "Observer-based sliding mode control for Itô stochastic time-delay systems with limited capacity channel," Journal of the Franklin Institute, vol. 349, no. 4, pp. 1602-1616, 2012.

[36] L. Wu and W. X. Zheng, "Passivity-based sliding mode control of uncertain singular time-delay systems," Automatica, vol. 45, no. 9, pp. 2120-2127, 2009.

[37] Q. Khan, A. I. Bhatti, M. Iqbal, and Q. Ahmed, "Dynamic integral sliding mode control for SISO uncertain nonlinear systems," International Journal of Innovative Computing, Information and Control, vol. 8, no. 7, pp. 4621-4633, 2012. 


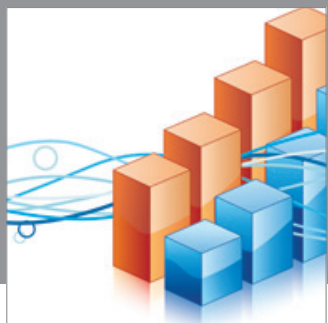

Advances in

Operations Research

mansans

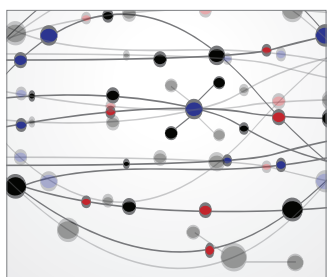

The Scientific World Journal
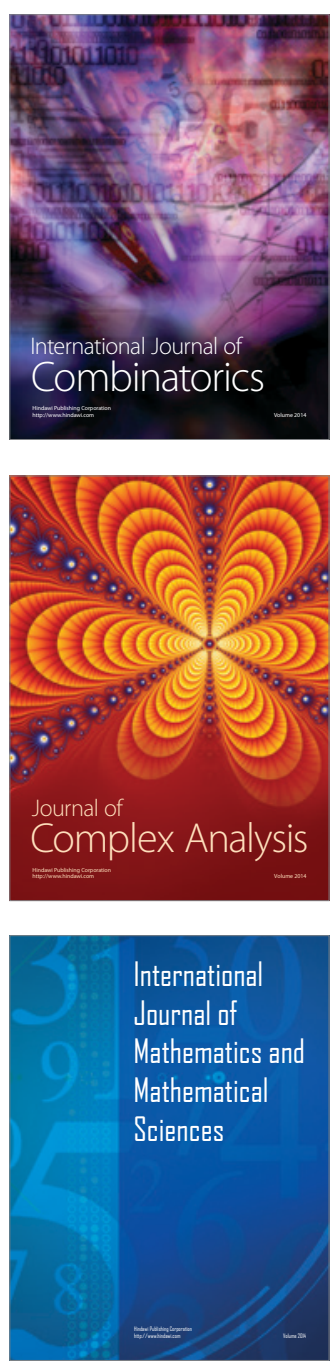
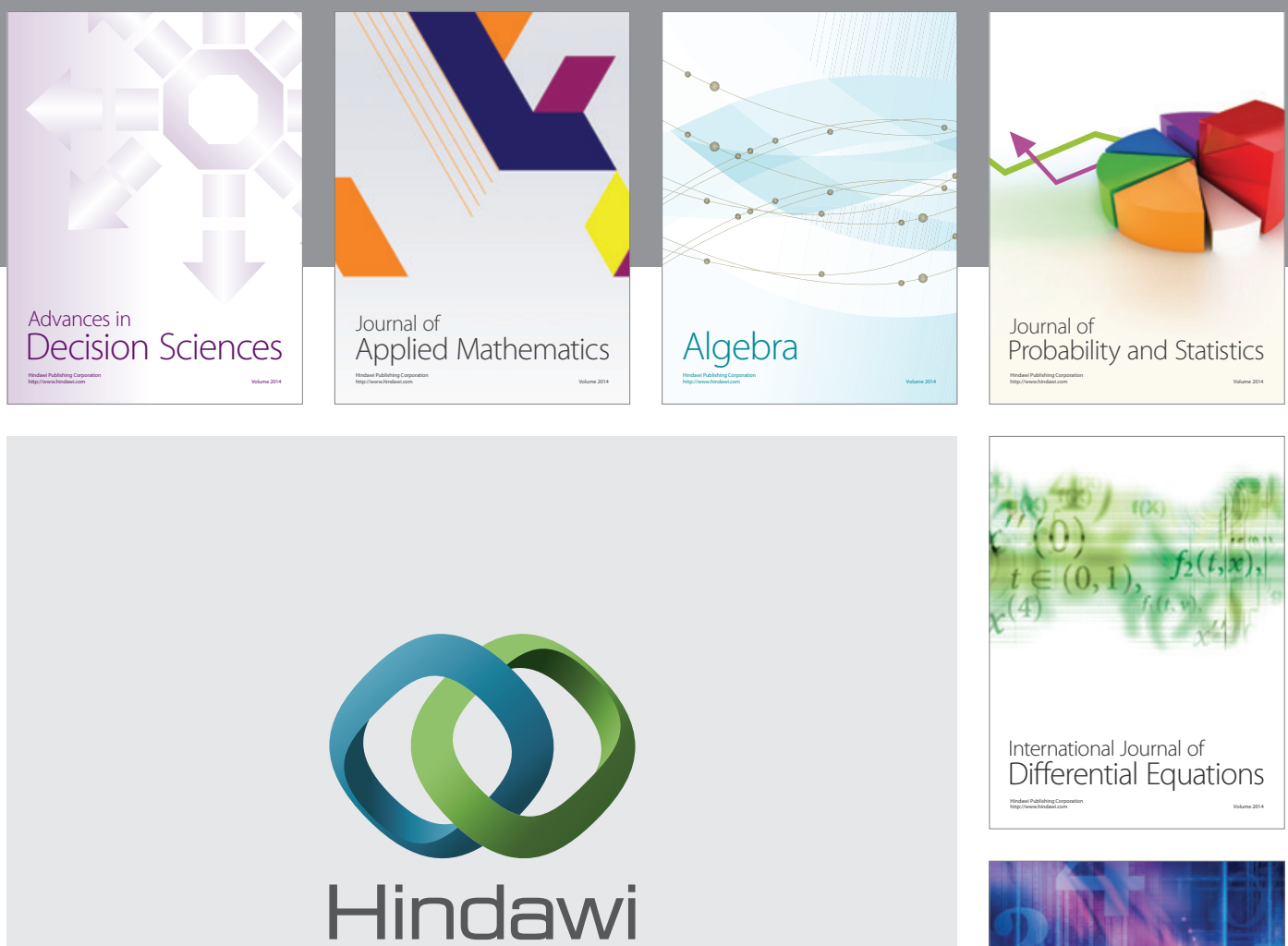

Submit your manuscripts at http://www.hindawi.com
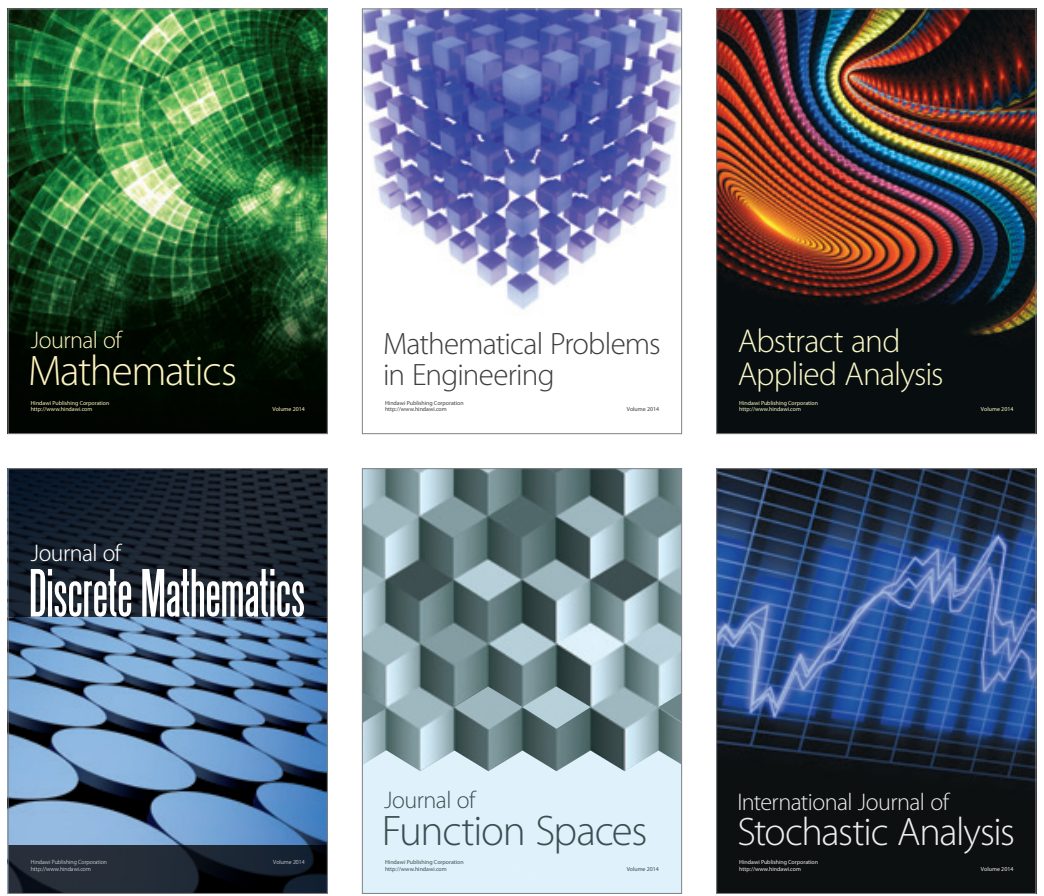

Journal of

Function Spaces

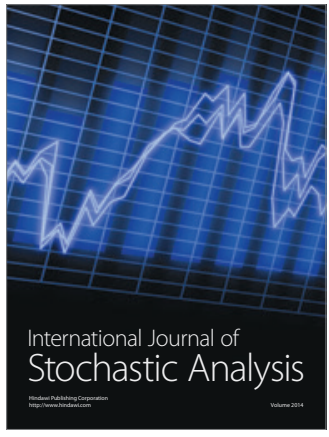

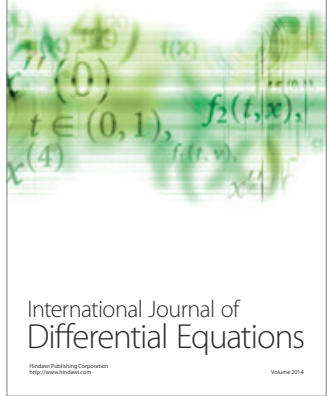
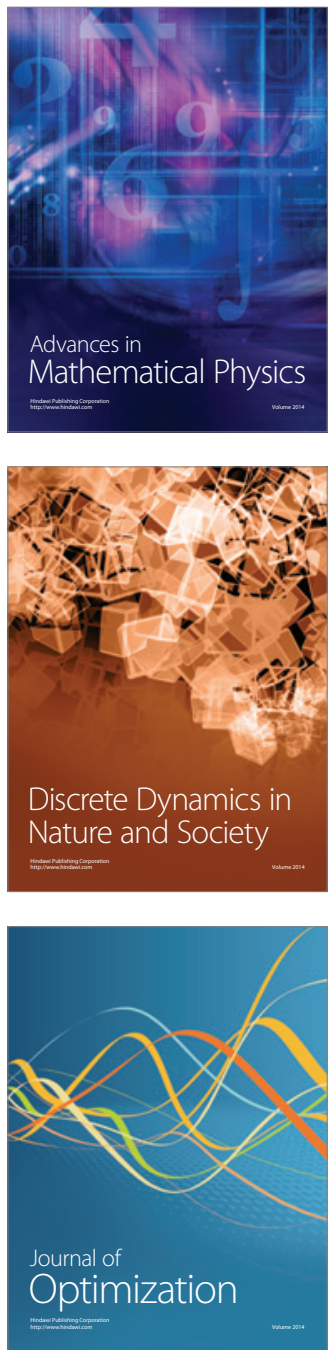